This item was submitted to Loughborough's Research Repository by the author.

Items in Figshare are protected by copyright, with all rights reserved, unless otherwise indicated.

\title{
On the recursive estimation of vehicular speed using data from a single inductance loop detector: a Bayesian approach
}

PLEASE CITE THE PUBLISHED VERSION

http://www.sciencedirect.com/science/article/pii/S0191261508000945

\section{PUBLISHER}

(C) Elsevier

\section{VERSION}

AM (Accepted Manuscript)

\section{LICENCE}

CC BY-NC-ND 4.0

\section{REPOSITORY RECORD}

Li, Baibing. 2019. "On the Recursive Estimation of Vehicular Speed Using Data from a Single Inductance Loop Detector: A Bayesian Approach". figshare. https://hdl.handle.net/2134/9157. 
This item was submitted to Loughborough's Institutional Repository (https://dspace.lboro.ac.uk/) by the author and is made available under the following Creative Commons Licence conditions.

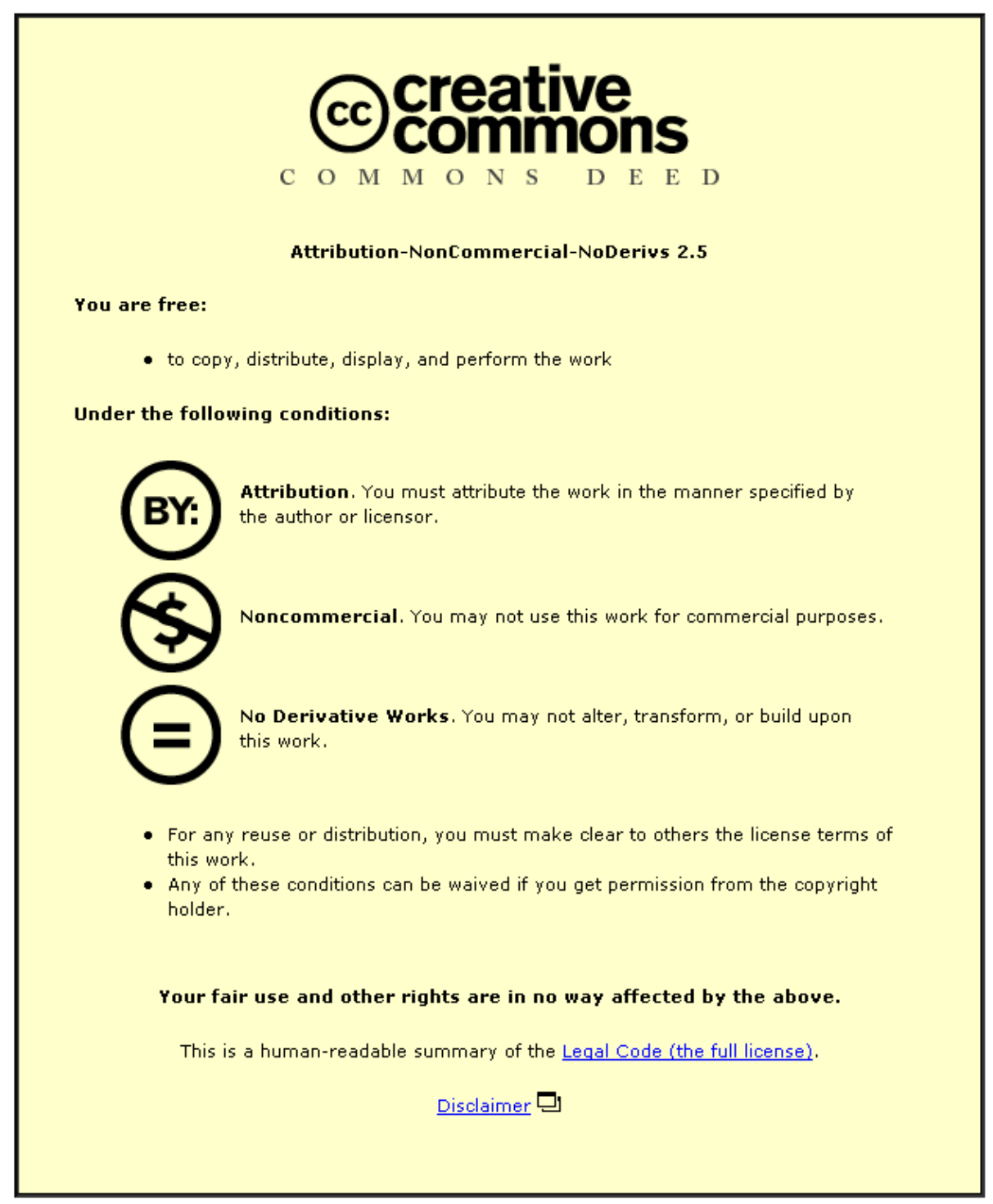

For the full text of this licence, please go to: http://creativecommons.org/licenses/by-nc-nd/2.5/ 


\title{
On the Recursive Estimation of Vehicular Speed \\ Using Data from a Single Inductance Loop Detector:
}

\section{A Bayesian Approach}

\author{
Baibing Li \\ Business School \\ Loughborough University \\ Loughborough LE11 3TU, United Kingdom \\ (email: b.li2@lboro.ac.uk)
}

\begin{abstract}
This paper investigates the recursive estimation of vehicular speed using the information provided by a single Inductance Loop Detector (ILD). A statistical model for space-mean speed measured by an ILD is developed, upon which a Bayesian analysis is carried out to estimate vehicular speed. This results in a set of recursive formulae which is analytically nice and neat. The incurred computational cost for updating the estimate of vehicular speed is kept to be a minimum. As a by-product, a simple method for the calibration of the effective vehicle length of an ILD is also developed. The proposed method is illustrated using simulation studies and a practical example.
\end{abstract}

Keywords: Bayesian analysis; Recursive estimation; Single inductance loop detector; Spacemean speed measurement; Vehicular speed 


\section{Introduction}

Traffic management relies on online collection and processing of information on traffic flow. Single Inductance Loop Detectors (ILDs) are cheap devices for collecting traffic data and thus are widely installed, playing a crucial role in online traffic controls. Data provided by an ILD include traffic volume $m_{k}$ and occupancy $O_{k}$ measured during each time interval $k$. They form the basis of various investigations on traffic flow such as speed estimation, accident analysis, etc. In recent years processing data collected from ILDs has been paid much attention in traffic engineering. This paper focuses on the issue of the estimation of vehicular speed using a pair of data $\left(O_{k}, m_{k}\right)$ collected via an ILD in each time interval $k$.

Most classical estimation methods of vehicular speed are based on the first-order method of moments approach, resulting in an estimator by the space-mean speed measurement $s_{k}$ which can be calculated using a pair of data $\left(O_{k}, m_{k}\right)$ collected via an ILD during time interval $k$ (see e.g. Kurkjian et al., 1980). These methods, however, lead to a biased estimator due to the replacement of the harmonic average by an arithmetic average (Hazelton, 2004). To address the issue of biasedness of the estimators, various methods have been developed to correct the biases. For instance Hall and Persaud (1989) have proposed to adjust an estimator by multiplying a correction constant. As Hall and Persaud (1989) have realised, however, the biases cannot be completely removed by a constant adjustment because the effect of the bias is not uniform.

In addition, the quality of the estimators derived by these methods is poor because they are based on a single piece of data, i.e. the space-mean speed measurement $s_{k}$ during time interval $k$. Statistically, pooling information obtained in successive time intervals can improve estimators. To address the issue of pooling information, Dailey (1999) has applied the Taylor's expansion to expand the space-mean speed measurement $s_{k}$ to the first two 
moments, resulting in a nonlinear function of the population speed parameter. This nonlinear function is linearised and is treated as the observation equation of a state space model. Dailey (1999) has then applied a standard technique, the Kalman filter, to estimate the population speed parameter. This approach produces a smooth estimate of vehicular speed by pooling information over successive time intervals. One consequence of these approximations, however, is that the resulting estimate is not robust in the sense that it greatly depends on the linearization (Ye et al. 2006). As demonstrated later in this paper, Dailey's method is sensitive to the choice of initial guess and/or changes in vehicular speed, where a slightly different choice of the initial point or an abrupt change in speed may lead the filter to completely break down. Recently, the approach of using Kalman filter method for vehicular speed estimation has been also considered by Ye et al. (2006) and Bickel et al. (2007).

To avoid approximations, Hazelton (2004) has performed Bayesian analysis and applied Markov Chain Monte Carlo (MCMC) to simulate the posterior distribution of vehicular speed, which has greatly improved on existing methods in terms of accuracy. This offline approach, however, is not practical in traffic controls. Due to the nature of traffic flow, an online estimation method is required in practice.

This paper contributes the literature of traffic engineering in two aspects. First, we investigate a statistical model for space-mean speed measurements. From a statistical point of view, the major difficulty in drawing statistical inference about vehicular speed lies in the fact that the distribution of space-mean speed measurements is analytically intractable under the normality assumption for individual speed measurements. In this paper, following Polus (1979), we assume that the traveling time required by a vehicle traversing a short road section has a gamma distribution. We then show that a space-mean speed measurement obtained by an ILD, $s_{k}$, follows an inverse gamma distribution. Secondly, we develop a set of recursive formulae via Bayesian analysis for fast estimation of vehicular speed. The recursive formulae 
are analytically nice and neat, incur a minimum computational cost, and can be easily implemented in practice. As a by-product, we also propose a simple method to calibrate the effective vehicular length of an ILD.

This paper is structured as follows. Section 2 is devoted to a statistical model for spacemean speed measurements. In Section 3, a Bayesian analysis is carried out to estimate vehicular speed recursively. Some practical issues raising when applying the recursive estimation method, including the calibration of the effective vehicular length, are investigated in Section 4. To illustrate the proposed method, simulation studies are examined in Section 5 and a practical example is investigated in Section 6. Finally concluding remarks are offered in Section 7. All proofs of theorems are given in the Appendix.

\section{A model for space-mean speed measurements}

\subsection{Notation}

Consider a single ILD that measures traffic flow during a time period that consists of a number of successive time intervals, each having a duration of $T$ (typically 20 to 30 seconds). In each time interval $k$, data measured by the ILD include the traffic volume $m_{k}$ (the count of vehicles) and the occupancy $O_{k}$ (the percentage of time that the ILD is occupied).

Now consider individual vehicles passing through the ILD. For the $j$ th vehicle passing through the ILD during time interval $k$, define

- $L_{k j}$ as the Effective Vehicle Length (EVL) associated with vehicle $j$ that is detectable by the ILD;

- $\quad t_{k j}$ as the time required by vehicle $j$ to travel the distance $L_{k j}$;

- $s_{k j}$ as the speed measurement of vehicle $j$ calculated as $s_{k j}=L_{k j} / t_{k j}$. 
It is conventional in traffic engineering to assume that the EVL is common to all vehicles which pass through an ILD during a time interval $k$, i.e. $L_{k j}=L_{k}$ for all $j$. In most of the studies, the common EVL $L_{k}$ is further assumed to be a constant parameter $L$ across different time intervals which is provided exogenously. Consequently, for a constant EVL parameter $L$, individual speed measurements reduce to

$$
s_{k j}=L / t_{k j} \quad\left(j=1, \ldots, m_{k}\right) .
$$

This latter assumption of the constant EVL parameter across different time intervals will be relaxed as a piecewise function of time in Section 4.

In traffic engineering the space-mean speed measurement $s_{k}$ during a time interval $k$ is defined to be the harmonic average of individual speed measurements $s_{k 1}, \ldots, s_{k m_{k}}$, i.e. $s_{k}=m_{k}\left\{\sum_{j=1}^{m_{k}} s_{k j}^{-1}\right\}^{-1}$. Let $v$ denote the population speed parameter which is the parameter that is to be estimated. Note that the space-mean speed $s_{k}$ is a biased estimate of the population speed parameter $v$ (Hall and Persaud, 1989; Hazelton, 2004).

Now substituting equations (2-1) and $\sum_{j=1}^{m_{k}} t_{k j}=T O_{k}$ into $s_{k}=m_{k}\left\{\sum_{j=1}^{m_{k}} s_{k j}^{-1}\right\}^{-1}$, the space-mean speed measurement can be calculated using the measurements $\left(O_{k}, m_{k}\right)$ collected from the ILD during time interval $k$ :

$$
s_{k}=m_{k} L /\left(T O_{k}\right)
$$

\subsection{A model for space-mean speed measurements from an ILD}

In this subsection we investigate a model for space-mean speed measurements. Let $\Gamma(\alpha, \beta)$ denote a gamma distribution having a probability density function $f(t)=\left\{\beta^{\alpha} / \Gamma(\alpha)\right\} t^{\alpha-1} \exp (-\beta t)$, where $\Gamma(\alpha)$ is the gamma function. In addition, let 
$\operatorname{in} \nu \Gamma(\alpha, \beta)$ denote an inverse gamma distribution having a probability density function $g(t)=\left\{\beta^{\alpha} / \Gamma(\alpha)\right\} t^{-(\alpha+1)} \exp (-\beta / t)$.

We first consider a special case where there is only one vehicle passing through the ILD during a time interval $k$, i.e. $m_{k}=1$. In this case, the space-mean speed measurement $s_{k}=m_{k}\left\{\sum_{j=1}^{m_{k}} s_{k j}^{-1}\right\}^{-1}$ reduces to the single speed measurement $s_{k 1}$. Polus (1979) has shown that the travelling time for a vehicle traversing a fixed distance approximately follows a gamma distribution, upon which the speed measured by the ILD can be characterised by the following theorem:

Theorem 1. Suppose that the travelling time $t_{k 1}$ required by a vehicle to traverse a distance of $L$ at a speed of $v$ follows a gamma distribution $\Gamma(\gamma, \gamma v / L)$ with an expected value of $L / v$ and a diffusion parameter $\gamma$. Then the speed $s_{k 1}=L / t_{k 1}$ measured by the ILD has an inverse gamma distribution $\operatorname{In} v \Gamma(\gamma, \gamma v)$.

We next turn to investigate the general situation where there is more than one vehicle passing through the ILD during a time interval $k$, i.e. $m_{k}>1$. For the space-mean speed measurement $s_{k}$ we have the following results:

Theorem 2. Let $s_{k 1}, \ldots, s_{k m_{k}}$ denote the unobservable speed measurements of $m_{k}\left(m_{k}>1\right)$ vehicles passing through an ILD during time interval $k$. If $s_{k j}$ have an inverse gamma distribution with a known diffusion parameter $\gamma$, i.e. $s_{k j} \mid v \sim \operatorname{Inv} \Gamma(\gamma, \gamma v)\left(j=1, \ldots, m_{k}\right)$, and conditional on $v$, the measurements $s_{k 1}, \ldots, s_{k m_{k}}$ are independent of each other, then 
(i) the space-mean speed measurement $s_{k}=m_{k}\left(\sum_{j=1}^{m_{k}} s_{k j}^{-1}\right)^{-1}$ also follows an inverse gamma distribution given by

$$
s_{k} \mid v \sim \operatorname{Inv} \Gamma\left(m_{k} \gamma, m_{k} \gamma v\right)
$$

(ii) the space-mean speed measurement $s_{k}=m_{k}\left(\sum_{j=1}^{m_{k}} s_{k j}^{-1}\right)^{-1}$ is a sufficient statistic for $v$.

We note that in many traffic studies, individual speed measurements $s_{k 1}, \ldots, s_{k m_{k}}$ derived from timing devices (e.g. stopwatches) over a fixed and short baseline are assumed to follow normal distributions (see e.g. Salter, 1989). Based on this assumption, the distribution of the space-mean speed measurement $s_{k}=m_{k}\left(\sum_{j=1}^{m_{k}} s_{k j}^{-1}\right)^{-1}$ is no longer analytically tractable. As a consequence, approximations are commonly used in practice to simplify problems related to space-mean speed measurements, see for instance, Daily (1999). Theorem 2 (i), in contrast, provides a simple statistical model to analyse space-mean speed measurements.

Under the assumption of a common EVL parameter, Theorem 2 (ii) further indicates that the aggregate signal $s_{k}$ has absorbed all useful information in $s_{k 1}, \ldots, s_{k m_{k}}$ in terms of drawing inference for vehicular speed $v$. Hence, no information is lost when it is the aggregate signal $s_{k}$, rather than the individual measurements $s_{k 1}, \ldots, s_{k m_{k}}$, that is observable.

\section{Bayesian analysis}

In this section, we perform a Bayesian analysis to investigate the recursive estimation of speed parameter $v$ using the collected space-mean speed measurement $s_{k}$ in a time interval $k$. 


\subsection{Bayesian inference during a time interval $k$}

Bayesian analysis combines two sources of information to draw inference about parameters of interest: (a) prior knowledge; and (b) the current observation on the parameters.

To draw statistical inference about the vehicular speed parameter $v$, we first consider the specification of the prior. In traffic engineering, normal and log-normal distributions, as well as gamma distributions are commonly used to model vehicular speed (Gerlough and Huber, 1975; Haight, 1963). The latter two types of distribution, log-normal and gamma, avoid the theoretical difficulty of negative speeds given by the left tails of normal distributions. In this paper, the prior distribution of vehicular speed $v$ during time interval $k$ is chosen as the following gamma distribution for mathematical convenience:

$$
v \sim \Gamma\left(\alpha_{k}, \alpha_{k} / \mu_{k-1}\right)
$$

where the prior mean is equal to $\mu_{k-1}$ and the prior standard deviation is equal to $\mu_{k-1} / \alpha_{k}^{1 / 2}$. The prior mean $\mu_{k-1}$ represents an estimate of the speed parameter $v$ obtained a priori. The hyper-parameter $\alpha_{k}$ reflects how accurate the prior information is about the vehicular speed parameter $v$. In general, the prior distribution is determined on the basis of the information collected in the previous time intervals. As shown later, because the prior and posterior distributions are conjugate, the same functional form of the prior distribution can be retained over successive time intervals. Further, the hyper-parameters of the prior distribution $\alpha_{k}$ and $\mu_{k-1}$ can be calculated recursively.

Now suppose that a space-mean speed measurement $s_{k}$ is available from the ILD during time interval $k$. According to Theorem 2, it follows an inverse gamma distribution given by equation (2-3). We can then apply Bayes' rule to combine the two sources of information: (a) the prior information (3-1); and (b) the current observation on the vehicular speed parameter 
$v$, i.e. the space-mean speed measurement $s_{k}$ in equation (2-3). The main result is summarised as follows:

Theorem 3. Suppose that a random variable $s$ given $v$ has an inverse gamma distribution $s \mid v \sim \operatorname{In} v \Gamma(\rho, \rho v)$ with a known parameter $\rho$. Then for the prior distribution $v \sim \Gamma(a, a / \mu)$, the posterior distribution is also a gamma distribution given by

$$
v \mid s \sim \Gamma\left(a+\rho, a \mu^{-1}+\rho s^{-1}\right) .
$$

From Theorem 3, given the space-mean speed measurement $s_{k}$, the posterior distribution of $v$ in time interval $k$ is also a gamma distribution:

$$
v \mid s_{k} \sim \Gamma\left(\alpha_{k}+m_{k} \gamma, \alpha_{k} \mu_{k-1}^{-1}+m_{k} \gamma s_{k}^{-1}\right) .
$$

On the basis of this posterior distribution, it is straightforward to obtain the posterior mean and posterior variance of the vehicular speed parameter $v$ :

$$
\begin{aligned}
& E\left(v \mid s_{k}\right)=\left\{\theta_{k} \mu_{k-1}^{-1}+\left(1-\theta_{k}\right) s_{k}^{-1}\right\}^{-1}, \\
& \operatorname{var}\left(v \mid s_{k}\right)=\mu_{k}^{2} /\left(\alpha_{k}+m_{k} \gamma\right),
\end{aligned}
$$

with a weight of $\theta_{k}=\alpha_{k} /\left(\alpha_{k}+m_{k} \gamma\right)$. In addition, a $(1-\alpha) \times 100 \%$ credible interval for $v$ is given by

$$
\left(\mu_{k} \chi_{1-\alpha / 2}^{2}\left(2\left(\alpha_{k}+m_{k} \gamma\right)\right) /\left\{2\left(\alpha_{k}+m_{k} \gamma\right)\right\}, \mu_{k} \chi_{\alpha / 2}^{2}\left(2\left(\alpha_{k}+m_{k} \gamma\right)\right) /\left\{2\left(\alpha_{k}+m_{k} \gamma\right)\right\}\right),
$$

where $\chi_{\alpha}^{2}(d f)$ is the value for the chi-squared distribution with $d f$ degrees of freedom that provides a probability of $\alpha$ to the right of the $\chi_{\alpha}^{2}(d f)$ value.

Denote the posterior mean as $\mu_{k}$. We use $\mu_{k}$ to estimate the population parameter $v$ in time interval $k$. It is a weighted harmonic average of $\mu_{k-1}$ and $s_{k}$ :

$$
\mu_{k}=\left\{\theta_{k} \mu_{k-1}^{-1}+\left(1-\theta_{k}\right) s_{k}^{-1}\right\}^{-1}
$$




\subsection{Recursive estimation}

Next, we turn to the issue of updating the estimate of the vehicular speed parameter $v$ when a new space-mean speed measurement $s_{k+1}$ becomes available in time interval $k+1$. Apparently, the prior information in time interval $k+1$ should be based on the posterior distribution obtained during time interval $k$. So from equation (3-2), the prior distribution in time interval $k+1$ can be taken as

$$
v \sim \Gamma\left(\alpha_{k+1}, \alpha_{k+1} / \mu_{k}\right)
$$

where

$$
\alpha_{k+1}=\alpha_{k}+m_{k} \gamma
$$

Note that the prior distribution (3-3) in time interval $k+1$ has the same functional form as equation (3-1). When a new observation of the space-mean speed $s_{k+1} \mid v \sim \operatorname{In} v \Gamma\left(m_{k+1} \gamma, m_{k+1} \gamma v\right)$ becomes available, the following posterior distribution in time interval $k+1$ can be obtained by applying Theorem 3 again:

$$
v \mid s_{k+1} \sim \Gamma\left(\alpha_{k+1}+m_{k+1} \gamma, \alpha_{k+1} \mu_{k}^{-1}+m_{k+1} \gamma s_{k+1}^{-1}\right) .
$$

Clearly, except for the subscript $k$ being replaced by $k+1$, it has the same form as equation (32). The updated estimate of the speed parameter $v$ is thus given by

$$
\begin{aligned}
& \theta_{k+1}=\alpha_{k+1} /\left(\alpha_{k+1}+m_{k+1} \gamma\right), \\
& \mu_{k+1}=\left\{\theta_{k+1} \mu_{k}^{-1}+\left(1-\theta_{k+1}\right) s_{k+1}^{-1}\right\}^{-1} .
\end{aligned}
$$

Consequently we can estimate vehicular speed recursively.

\subsection{Forgetting factor}

In practice, however, vehicular speed may evolve slowly over time. To take this into account, a standard practice in Bayesian statistics for utilizing information obtained 
previously or obtained elsewhere is to inflate the variance of the posterior distribution obtained in the past when it is treated as the prior distribution in the current analysis, reflecting the fact that we are less sure about the current value of the parameter (see e.g. Congdon, 2001). To put this in another word, we may define a 'forgetting factor' so that observations are weighed differently when they are used to estimate a parameter, where the latest observation weighs highest. This is a commonly used approach in the algorithms of recursive estimation (see e.g. Ljung and Soderstrom, 1987).

Specifically, instead of equations (3-3) and (3-4), the prior distribution incorporated in time interval $k+1$ is now taken as

$$
v \sim \Gamma\left(\delta\left(\alpha_{k}+m_{k} \gamma\right), \delta\left(\alpha_{k} \mu_{k-1}^{-1}+m_{k} \gamma s_{k}^{-1}\right)\right)
$$

where $\delta(0<\delta<1)$ is a forgetting factor. As demonstrated in $\mathrm{Li}(2005)$, the forgetting factor $\delta$ in equation (3-6) does not affect the mean so that the prior mean in time interval $k+1$ is still equal to $\mu_{k}$. However, the prior standard deviation in equation (3-6) becomes to $\mu_{k} /\left\{\delta\left(\alpha_{k}+m_{k} \gamma\right)^{1 / 2}\right\}$ which is inflated since $0<\delta<1$. In practice, the forgetting factor is usually treated as a tuning parameter (see e.g. Ljung and Soderstrom, 1987).

Now let

$$
\alpha_{k+1}=\delta\left(\alpha_{k}+m_{k} \gamma\right) \quad \text { for } k=1,2, \ldots
$$

so that the prior distribution can be rewritten as $v_{k+1} \sim \Gamma\left(\alpha_{k+1}, \alpha_{k+1} / \mu_{k}\right)$. The posterior distribution in time interval $k+1$ still has the same functional form as equation (3-5) but $\alpha_{k+1}$ is now updated as (3-7) rather than (3-4). The algorithm for the recursive estimation of vehicular speed is summarised as follows:

\section{ALGORITHM.}


Given: Tuning parameter $\delta$; Diffusion parameter $\gamma$; Initial estimates $\mu_{0}$ and $\alpha_{0} ; m_{0}=0$; EVL parameter $L$; Constant coefficient $T$.

For $k=1: K$

Step 1. Collect a pair of data $\left(O_{k}, m_{k}\right)$;

Step 2. Calculate the space-mean speed measurement $s_{k}=m_{k} L /\left(T O_{k}\right)$;

Step 3. Calculate $\alpha_{k}=\delta\left(\alpha_{k-1}+m_{k-1} \gamma\right)$ and $\theta_{k}=\alpha_{k} /\left(\alpha_{k}+m_{k} \gamma\right)$;

Step 4. Estimate the speed parameter $v$ as $\mu_{k}=\left\{\theta_{k} \mu_{k-1}^{-1}+\left(1-\theta_{k}\right) s_{k}^{-1}\right\}^{-1}$;

End.

It should be noted that in practice, there may not be any vehicles passing through an ILD during a particular time interval $k$. In this case, the posterior distribution can be simply taken the same as the prior distribution, i.e. $\mu_{k}=\mu_{k-1}$ and $\alpha_{k}=\delta \alpha_{k-1}$.

In addition, we note that when the chosen forgetting factor $\delta$ is sufficiently small, the weight $\theta_{k}$ will be very close to zero. Consequently the estimated speed $\mu_{k}=\left\{\theta_{k} \mu_{k-1}^{-1}+\left(1-\theta_{k}\right) s_{k}^{-1}\right\}^{-1}$ reduces to the first-order method of moments approach.

\subsection{One-step-ahead forecasting}

For a given number of vehicles passing through the ILD, $m_{k+1}$, during time interval $k+1$, we can predict the space-mean speed measurement $s_{k+1}$ on the basis of the information collected in time interval $k$.

Theorem 4. The one-step-ahead predictive distribution of $s_{k+1} / \mu_{k}$ obtained in time interval $k$ has an $F$ distribution with degrees of freedom $2 \alpha_{k+1}$ and $2 m_{k+1} \gamma$ respectively. The predicted 
value of $s_{k+1}$, taken as the mean of the predictive distribution, is $\hat{s}_{k+1}=m_{k+1} \gamma \mu_{k} /\left(m_{k+1} \gamma-1\right)$. A $(1-\alpha) \times 100 \%$ credible interval for $s_{k+1}$ is

$$
\left(\mu_{k} F_{1-\alpha / 2}\left(2 \alpha_{k+1}, 2 m_{k+1} \gamma\right), \mu_{k} F_{\alpha / 2}\left(2 \alpha_{k+1}, 2 m_{k+1} \gamma\right)\right)
$$

where $F_{\alpha}(a, b)$ is the value for the $F$ distribution with degrees of freedom $a$ and $b$ that provides a probability of $\alpha$ to the right of the $F_{\alpha}(a, b)$ value.

\section{Some practical issues}

In this section we discuss some practical issues raising when applying the recursive estimation method developed in the previous section.

\subsection{Estimation of the diffusion parameter $\gamma$}

For the recursive formulae developed in the previous section it is assumed that the diffusion parameter $\gamma$ is a known parameter. In practice, this diffusion parameter has to be estimated from historical data before the recursive method is applied.

The method of moments can be used to estimate $\gamma$ as follows. Suppose that we have collected some data from an ILD, $\left(O_{k}, m_{k}\right)(k=1, \ldots R)$, under the circumstance that vehicular speed is approximately uniform. Define $u_{k}=m_{k} / T O_{k}$ to be a scaled space-mean speed measurement such that $L u_{k}$ is a space-mean speed. Then we have $u_{k} \mid v \sim \operatorname{In} v \Gamma\left(m_{k} \gamma, m_{k} \gamma v / L\right)$. Let $h_{k}=u_{k}^{-1}$. Then we have $h_{k} \mid v \sim \Gamma\left(m_{k} \gamma, m_{k} \gamma v / L\right)$ with $E h_{k}=L / v$ and $\operatorname{var}\left(h_{k}\right)=(L / v)^{2}\left(m_{k} \gamma\right)^{-1}$. Let $\bar{h}$ and $\hat{\sigma}^{2}$ denote the sample mean and sample variance of $h_{k}$ respectively $(k=1, \ldots R)$. It is easy to verify that

$$
E \bar{h}=L / v \quad \text { and } \quad E \hat{\sigma}^{2}=(L / v)^{2} \gamma^{-1} \sum_{k=1}^{R} m_{k}^{-1} /(R-1)
$$


Consequently, we obtain $(E \bar{h})^{2} / E \hat{\sigma}^{2}=\gamma /\left\{\sum_{k=1}^{R} m_{k}^{-1} /(R-1)\right\}$ which is not related to the parameters $v$ and $L$. Hence, the diffusion parameter $\gamma$ can be estimated as $\hat{\gamma}=\left(\bar{h}^{2} / \hat{\sigma}^{2}\right) \sum_{k=1}^{R} m_{k}^{-1} /(R-1)$.

\subsection{Initial prior distribution}

In the algorithm outlined in the previous section, the parameters $\mu_{0}$ and $\alpha_{0}$ of the initial prior distribution in time interval $k=1$ need to be specified.

A non-informative prior in time interval $k=1$ can be incorporated, where $\alpha_{0}$ is taken sufficiently small, say $10^{-6}$, and $\mu_{0}$ can be taken as any reasonable value between 0 to 100 , resulting in a sufficiently large prior variance. Consequently, in time interval $k=1$, the weight $\theta_{1}$ is close to zero and $\mu_{1}=\left\{\theta_{1} \mu_{0}^{-1}+\left(1-\theta_{1}\right) s_{1}^{-1}\right\}^{-1} \approx s_{1}$. Note that this is also the approach incorporated in the recursive least squares estimation (see e.g. Ljung and Soderstrom, 1987).

One advantage of using the method proposed in this paper is that it is not sensitive to the choice of initial estimates as long as the prior variance is taken sufficiently large.

\subsection{Estimation of the EVL parameter via calibration}

The value of the EVL parameter $L$ is usually determined using exogenous data collected from a comparable type of road (Dailey, 1999; Hazelton, 2004). In this subsection, we suggest an alternative method for the estimation of the EVL parameter.

First we note that it is unrealistic to assume that the EVL parameter is constant across different time intervals. In practice, the EVL parameter may vary during the time of day. For instance, in the earlier morning or late evening road trains and trucks are usually more prevalent so that the EVL parameter may have a larger value. 
The assumption of the constant EVL parameter is relaxed as follows. First, we separate peak hours from the rest of time by dividing a whole day of time into several time periods and treat the EVL as a piecewise function of time within each of these time periods.

Now we focus on one particular time period and consider the calibration of the EVL. Suppose that a random sample of vehicular speed data, $\left\{z_{k}\right\}(k=1, \ldots, M)$, is available in $M$ time intervals via a temporally installed speedmeter, subject to some measurement errors $e_{k}$ with a zero-mean. In addition, suppose measurements $\left(O_{k}, m_{k}\right)(k=1, \ldots, M)$ have been collected via an ILD during the same time period. As defined in Section 4.1, let $u_{k}=m_{k} / T O_{k}$ be a scaled space-mean speed measurement. Applying the recursive formulae in Section 3 to the scaled space-mean speed measurements $u_{k}$, we can obtain a sequence of estimates of vehicular speed $\left\{x_{k}\right\}$, up to a proportional constant $L$. Then the value of the EVL parameter $L$ may be estimated by solving the following least squares problem, $\min _{L} \sum_{k=1}^{M}\left\{z_{k}-L x_{k}\right\}^{2}$, which yields an estimate of the EVL parameter, $\hat{L}=\sum_{k=1}^{M} z_{k} x_{k} / \sum_{k=1}^{M} x_{k}^{2}$.

If a more accurate estimate is required, the EVL can be treated as a piecewise linear function of time, i.e. $L=c_{0}+c_{1} k$ within each time period, where $k$ represents the $k$ th time interval in the time period. $c_{0}$ and $c_{1}$ are two coefficients to be determined. We further require that the piecewise linear function is continuous over the whole time period of interest. Mathematically this imposes some equality constraints at boundary points of each time period since EVL expressions in two adjacent time periods are equal at the common boundary point. The EVL function can be estimated by solving a least squares problem to minimize the observed and calculated speeds, subject to the continuity constraints.

Other time dependent, location specific vehicle length estimation methods have also been proposed; see Jain and Coifman (2005) and references therein. 


\subsection{Choice of the forgetting factor}

The optimal value for the forgetting factor $\delta$ depends on many other factors such as the level of vehicle congestion, etc. In practice, it is usually treated as a tuning parameter so that it is determined experimentally.

In the case where measured speed data are available via a temporarily installed speedmeter, the forgetting factor can be calculated as follows. Consider a grid of points between 0 and 1 that $\delta$ may take, say from $\delta_{\min }$ to $\delta_{\max }$ by a step of $\tilde{\delta}\left(0<\delta_{\min }<\delta_{\max }<1\right)$. For each point of $\delta$, we apply the algorithm in Section 3 to estimate vehicular speed. We then compare the estimated speed with the measured speed. The forgetting factor is chosen as the one which leads to the minimum mean squared error.

\section{Simulation studies}

In this section, we examine two numerical examples to illustrate the developed method. One major advantage of carrying out simulation studies is that 'true' values of vehicular speed are known a priori so that it is straightforward to assess the performance of an estimation method in terms of accuracy.

\subsection{Simulation study $I$}

In this subsection a simulation study is carried out to illustrate how the developed method works under the assumptions outlined in Sections 2 and 3.

\subsubsection{Data generation}

Consider an ILD that provides measurements of traffic volume and occupancy during a number of time intervals of duration 20 seconds. The EVL parameter is assumed to be a constant and set as $L=24$ feet. Traffic flow was simulated in 1000 time intervals. To 
accommodate the nature that vehicular speed evolves slowly over time, 'true' values of vehicular speed were simulated using a random walk having an initial speed of $60 \mathrm{mph}$ and a standard deviation of $1 \mathrm{mph}$. The number $m_{k}$ of vehicles passing through the ILD was simulated using a Poisson distribution having a mean of 4 . The time required by each vehicles to pass through the ILD was simulated from $\Gamma(\gamma, \gamma \nu / L)$ with $\gamma=15$, where $v$ was the speed simulated above.

\subsubsection{Recursive estimation with a known $L$}

We first assumed that the EVL parameter of $L=24$ feet was known a priori. An initial prior representing vague information about vehicular speed was incorporated with $\mu_{0}=50$ and $\alpha_{0}=10^{-6}$. The forgetting factor was set as $\delta=0.8$. Because $\alpha_{0}$ was set to be small, the initial prior had little impact on the subsequent recursive estimation.

The traffic data in the first 200 time intervals were considered as historical data and used to estimate the diffusion parameter $\gamma$. The traffic data in the remaining 800 time intervals were used to estimate vehicular speed. Figure 1a displays the estimates of vehicular speed using $\mu_{k}$ (dotted line). For comparison the 'true' values of vehicular speed are also superimposed (real line). It can be seen that the estimates of vehicular speed have captured most variation of the 'true' values. Figure $1 \mathrm{~b}$ displays the envelop of a nominal $95 \%$ credible intervals (the dotted lines), where the 'true' values of vehicular speed are also plotted (real line) on the same graph. In total there were $2.13 \%$ time intervals during which the 'true' values lied outside the nominal $95 \%$ credible intervals.

Figure 1a. The 'true' values of vehicular speed (real line) and the estimated values by the recursive method (dotted line). 
Figure 1b. The envelop of a nominal 95\% credible intervals (dotted lines) and the 'true' values of vehicular speed (real line).

\subsubsection{Recursive estimation with an unknown $L$}

We next turn to investigate the impact when the value of the EVL parameter $L$ is unknown. Suppose that speed data were available, subject to some errors, via a temporarily installed speedmeter in the first 200 time intervals. These measured speed data were generated in the simulation study as the 'true' values of speed plus a normal random noise having a zero-mean and a standard deviation of $2 \mathrm{mph}$.

Applying the calibration method in Section 4.3, we obtained an estimated value of $L$. The recursive estimation method was then applied to analyse the data in the remaining 800 time intervals. The root mean squared error (RMSE) between the estimated values and the corresponding 'true' values of vehicular speed was calculated, $2.25 \mathrm{mph}$. They are comparable to the RMSE obtained using the 'true' value of the EVL parameter, $2.15 \mathrm{mph}$.

\subsubsection{The impact of the choice for the forgetting factor}

It is of interest to investigate the impact of the forgetting factor on the accuracy of estimation. Table 1 displays the estimation errors in terms of RMSE when the forgetting factor $\delta$ takes a value from 0.60 to 0.95 by a step of 0.05 . It can be seen that the errors are comparable to each other unless the forgetting factor becomes too large/small. For this particular data set, a value of the forgetting factor between 0.75 to 0.85 is a good choice.

In practice, the forgetting factor is usually treated as a tuning parameter. A suitable value may be determined experimentally using the method in Section 4.4. 
(Table 1)

\subsubsection{Repeated experiments}

Finally we assess the developed method via repeated simulation experiments, where the diffusion parameter $\gamma$ is taken as 15 and 25 respectively, and the value of the forgetting factor $\delta$ is determined using the method in Section 4.4. Totally 30 experiments were conducted. The resulting RMSEs averaged over the 30 experiments are displayed in Table 2.

(Table 2)

From Table 2 we see that the developed method has a better performance than that of the classical method where vehicular speed is estimated using the current observation of spacemean speed $s_{k}$ (Kurkjian et al., 1980). This is not surprising because as pointed out earlier, the classical method may be considered as a special case of the developed method where the forgetting factor is small. Consequently, by tuning the forgetting factor, the developed recursive method can always produce a better estimate. The developed method will become significantly superior to the classical method when measurements of space-mean speed are very noisy due to measurement errors. In this case a smoothed estimate can greatly improve the quality of estimation by pooling information collected over successive time intervals.

It can also be seen from Table 2 that the estimates of vehicular speed obtained without knowing the 'true' value of the EVL parameter were only slightly worse than that obtained with the known 'true' value of the EVL parameter.

\subsection{Simulation study II}


We next turn to draw a numerical comparison with Dailey's method (1999). The Dailey's method is based on the Kalman filter, where the system equation which characterises the evolution of vehicular speed over the time is assumed to be an $\operatorname{AR}(2)$ process, $v_{k}=a v_{k-1}+b v_{k-2}+w_{k}$, and the observation equation is $O_{k} / m_{k}=(L / T)\left\{\left(\sigma_{s}^{2}+v_{k}^{2}\right) / v_{k}^{3}\right\}+\varepsilon_{k}$, where $a$ and $b$ are two coefficients, $w_{k}$ and $\varepsilon_{k}$ are two independent error terms having zeromeans and standard deviations of $\sigma_{s}$ and $\sigma_{O / N}$ respectively.

\subsubsection{Data generation}

Data were generated according to the parameters used in Dailey (1999). The values of 'true' vehicular speed were simulated as an $\operatorname{AR}(2)$ process, $v_{k}=0.7837 v_{k-1}+0.2104 v_{k-2}+w_{k}$, with $w_{k} \sim N\left(0, \sigma_{s}^{2}\right)$ and $\sigma_{s}=14$. The initial speed was set as $60 \mathrm{mph}$. The EVL parameter was set as $L=22$ feet. The number of vehicles $m_{k}$ was generated as an outcome of a Poisson variable with a mean of 8.775 . Following Hazelton (2004), the measured time by the ILD during time interval $k$ was simulated as $t_{k}=\left(m_{k} L / v_{k}\right)\left(1+0.05 z_{k}\right)$ with $z_{k} \sim N(0,1)$. As shown later, this resulted in an average estimate of $\sigma_{O / N}$ equal to 0.00040 whose magnitude was in line with that of 0.00078 used in Dailey (1999).

\subsubsection{Speed estimation using Dailey's method}

For Dailey's method to have a full power, the data used to identify the system equation was re-used to estimate vehicular speed. Specifically, the simulated speed data and loop data were used to estimate the coefficients $a$ and $b$ of the AR(2) process as well as $\sigma_{s}$ and $\sigma_{O / N}$. Then the Kalman filter was applied to estimate vehicular speed using the loop data only, where the initial speed used for simulating speed data, $60 \mathrm{mph}$, was incorporated as the initial guess of the Kalman filter. The values of the estimated speed were compared to the 'true' 
values of the simulated speed, and the error in terms of RMSE was calculated. The experiments were repeated 30 times.

The estimated parameters by Dailey's method were close to their corresponding true values. The averages of the estimated parameters over the 30 experiments were $\hat{a}=0.7586$, $\hat{b}=0.2290, \hat{\sigma}_{s}=13.8778$ and $\hat{\sigma}_{O / N}=0.00040$ respectively. The resulting value of RMSE is displayed in Table 3.

Next $\sigma_{s}$ was re-set as $10 \mathrm{mph}$ and $5 \mathrm{mph}$ respectively and the experiments were conducted in the same manner as outlined earlier. The averages of the estimated parameters were $\hat{a}=0.7519, \hat{b}=0.2421, \hat{\sigma}_{s}=9.7559$ and $\hat{\sigma}_{O / N}=0.00032$ when $\sigma_{s}=10$; whereas the averages of the estimated parameters were $\hat{a}=0.8001, \hat{b}=0.1962, \hat{\sigma}_{s}=5.0513$ and $\hat{\sigma}_{O / N}=$ 0.00035 when $\sigma_{s}=5$. The resulting values of RMSE are also displayed in Table 3 .

\subsubsection{A comparison}

For comparison, the recursive method developed in this paper was also applied to estimate the vehicular speed as follows. First the simulated data were used to estimate the coefficient $\gamma$. The optimal value of the forgetting factor $\delta$ was determined using the method in Section 4.4. The values of the estimated speed by the developed recursive method were compared to the 'true' values of the simulated speed, and the error in terms of RMSE was calculated and displayed in Table 3.

It can be seen from Table 3 that Dailey's method has higher estimation errors than the developed method due to the approximations made in Dailey's method.

(Table 3) 
Finally before concluding this section, we note that as a consequence of linearisation, the Dailey's method was sensitive to the choice of initial speed. During our simulation study, it was not unusual that the Kalman filter completely broke down (i.e. greatly deviated from the trajectory of the 'true' vehicular speed) even if the initial speed of $60 \mathrm{mph}$ used in data generation was incorporated as the starting point of the filter. All the cases where the Kalman filter broke down were excluded from the reported results in Table 3.

\section{A practical example}

In this section we present an empirical analysis to real traffic flow data. To evaluate the performances of different methods, we follow Dailey's and Hazelton's approaches and compare the estimated vehicular speed with the measured speed data obtained by a speed trap located close to a selected ILD. Although the measured speed data are not noise-free, they provide a reference point about the values of vehicular speed with which the estimated values of vehicular speed can be compared.

\subsection{Data}

The data were downloaded from the database managed by Traffic Data Acquisition and Distribution (TDAD) project by the Intelligent Traffic Systems group at the University of Washington at http://www.its.washington.edu/tdad. The downloaded data were recorded between 5:00 am and 10:00 am on Thursday, May 10, 2007, at a site in Interstate 5. Figure 2a displays the space-mean speed measurements $s_{k}$ calculated from the ILD detector.

\subsection{Data analysis}


First, the data collected during 5:00 am to 6:00 am were considered as historical data used to estimate the diffusion parameter $\gamma$. With the speed data measured via the speed trap during 5:00 am to 6:00 am, the constant EVL parameter was also calibrated.

Then we applied the recursive estimation method to estimate vehicular speed of traffic flow during 6:00 am to 10:00 am. We used the same vague initial prior as in the simulation study, i.e. $\mu_{0}=50$ and $\alpha_{0}=10^{-6}$, and the forgetting factor was set as $\delta=0.8$. Figure $2 \mathrm{~b}$ displays the estimated vehicular speed (real line). For comparison, the speed data measured by the speed trap are also superimposed (dotted line). Note that there were some missing values in the measured speed data which were replaced by their adjacent speed measurements when plotting Figure 2b. Overall, it can be seen that the developed method has done a good job in terms of reproducing the measured vehicular speed. Also, as shown in Figure $2 b$, the recursive estimation method provides a quite smooth estimate of vehicular speed. In contrast the measurements of vehicular speed via the speed trap were very noisy.

Figure 2a. The observations of space-mean speed.

Figure $2 b$. The measured vehicular speed by a speed trap (dotted line) and the estimated vehicular speed by the developed method (real line).

\subsection{Analysis using Dailey's method}

On the basis of the historical loop data measured by the ILD and the speed data measured by the speed trap during 5:00 am to 6:00 am, the parameters in Dailey method were estimated.

We then applied Dailey's method to estimate vehicular speed of traffic flow during 6:00 am to 10:00 am, where the same value of the EVL parameter and the same initial value of 
speed used in Section 6.2 were incorporated. Figure 2c displays the estimated vehicular speed (real line). For comparison, the speed data measured by the speed trap are also superimposed (dotted line). Overall, it can be seen that Dailey's method performed well until about 9 am. Shortly after 9:00 am, however, the speed of the traffic flow suddenly reduced and this abrupt change caused the Dailey's method broke down due to the linearization of the non-linear observation equation in Dailey's method, as shown in Figure 2c.

Figure $2 c$. The measured vehicular speed by a speed trap (dotted line) and the estimated vehicular speed by Dailey' method (real line).

\section{Concluding remarks}

In this paper, we have investigated the recursive estimation of vehicular speed using the data collected from a single ILD. A recursive estimation method has been developed. The resulting recursive formulae have a nice analytical form, and the incurred computational cost is kept to be a minimum. As a by-product, a simple calibration method is developed to estimate the effective vehicle length.

This proposed recursive estimation method includes the first-order method of moments approach as its special case where the forgetting factor is chosen as a small value. It is thus not surprising that it has a better numerical performance than the classical method. In comparison with Dailey's method, it is more reliable where the linearization used in Dailey's method is avoided. In addition, there is no need for this method to identify a dynamic system equation as required in Dailey's method.

We have also developed a statistical model for space-mean speed measurements. Spacemean speed plays an important role in traffic engineering. As a harmonic average of 
individual speed measurements, however, it is hard to deal with space-mean speed analytically under the normality assumption for individual speed measurements. In this paper we have developed a model for space-mean speed measurements which possesses some very nice theoretical properties and can be applied in a wider areas in traffic engineering.

\section{Acknowledgement}

The author would like to thank the referee for his/her valuable comments on earlier versions of this paper.

\section{Appendix. Proofs of theorems}

Proof of Theorem 1. According to the definition of inverse gamma distributions, $t_{k 1} \sim \Gamma(\gamma, \gamma \nu / L) \Leftrightarrow 1 / t_{k 1} \sim \operatorname{inv} \Gamma(\gamma, \gamma \nu / L)$. Since $L$ is a constant, we obtain $s_{k 1} \sim \operatorname{In} v \Gamma(\gamma, \mathcal{W})$.

Proof of Theorem 2. (i) $s_{k j} \mid v \sim \operatorname{Inv} \Gamma(\gamma, \gamma v)$ if and only if $s_{k j}^{-1}$ has a gamma distribution, $\Gamma(\gamma, \gamma v)$. Since $\sum_{j=1}^{m_{k}} s_{k j}^{-1} / m_{k} \sim \Gamma\left(m_{k} \gamma, m_{k} \gamma v\right)$, we obtain $s_{k} \sim i n v \Gamma\left(m_{k} \gamma, m_{k} \gamma v\right)$.

(ii) Let $p\left(s_{k j} \mid v\right)$ denote the density function of $\operatorname{Inv} \Gamma(\gamma, \gamma v)$. We note that the joint distribution of $s_{k 1}, \ldots, s_{k m_{k}}, p\left(s_{k_{1}}, \ldots, s_{k_{m}} \mid v\right)=\prod_{j=1}^{m_{k}} p\left(s_{k_{j}} \mid v\right)$, is given by

$$
\left[\gamma^{m_{k} \gamma} /\{\Gamma(\gamma)\}^{m_{k}}\right]\left(\prod_{j=1}^{m_{k}} s_{k_{j}}\right)^{-(\gamma+1)} \exp \left\{-\gamma \sum_{j=1}^{m_{k}} s_{k_{j}}^{-1}\right\}
$$

From part (i) we know that the density $p\left(s_{k} \mid v\right)$ is $\operatorname{In} v \Gamma\left(m_{k} \gamma, m_{k} \gamma v\right)$. Hence the conditional distribution of $s_{k 1}, \ldots, s_{k m_{k}}$ given $s_{k}, p\left(s_{k_{1}}, \ldots, s_{k_{m}} \mid v\right) / p\left(s_{k} \mid v\right)$, can be shown to be 


$$
\left[m_{m}{ }^{m_{k} \gamma} \Gamma\left(m_{k} \gamma\right) /\{\Gamma(\gamma)\}^{m_{k}}\right] s_{k}^{\left(m_{k} \gamma+1\right)} \prod_{j=1}^{m_{k}} s_{k_{j}}{ }^{-(\gamma+1)},
$$

which does not depend on $v$. Hence, $s_{k}$ is a sufficient statistic for $v$. This completes the proof.

Proof of Theorem 3. Let $p(v)$ and $p(s \mid v)$ denote the prior distribution $\Gamma(a, a / \mu)$ and observation distribution $\operatorname{In} v \Gamma(\rho, \rho v)$ respectively. Applying Bayes' rule, the posterior distribution of the parameter $v$ is given by:

$$
\begin{aligned}
& p(v \mid s) \propto p(v) \times p(s \mid v) \\
& \propto v^{a-1} \exp (-a v / \mu) \times v^{\rho} \exp (-\rho v / s) \\
& =v^{a+\rho-1} \exp \{-(a / \mu+\rho / s) v\} .
\end{aligned}
$$

Hence, the posterior distribution of the parameter $v$ is a gamma distribution, i.e. $v \mid s \sim \Gamma\left(a+\rho, a \mu^{-1}+\rho s^{-1}\right)$. This completes the proof.

Proof of Theorem 4. Let $p\left(s_{k+1} \mid v\right)$ and $p\left(v \mid s_{k}\right)$ denote the distribution of the space-mean speed measurement and the prior distribution in time interval $k+1$. They are given by $\operatorname{In} v \Gamma\left(m_{k+1} \gamma, m_{k+1} \gamma v\right)$ and $\Gamma\left(\alpha_{k+1}, \alpha_{k+1} / \mu_{k}\right)$ respectively. The one-step-ahead predictive distribution is

$$
\begin{aligned}
& p\left(s_{k+1} \mid s_{k}\right)=\int_{-\infty}^{+\infty} p\left(s_{k+1} \mid v\right) p\left(v \mid s_{k}\right) d v \\
& =A_{1} s_{k+1}^{-\left(m_{k+1} \gamma+1\right)} \mu_{k}^{-\alpha_{k+1}} \int_{-\infty}^{+\infty} v^{m_{k+1} \gamma+\alpha_{k+1}-1} \exp \left\{-\left(m_{k+1} \gamma s_{k+1}^{-1}+\alpha_{k+1} \mu_{k}^{-1}\right) v\right\} d v,
\end{aligned}
$$

where $A_{1}=\left(m_{k+1} \gamma\right)^{m_{k+1} \gamma} \alpha_{k+1}^{\alpha_{k+1}} /\left\{\Gamma\left(\alpha_{k+1}\right) \Gamma\left(m_{k+1} \gamma\right)\right\}$. Since

$$
\int_{-\infty}^{+\infty} v^{m_{k+1} \gamma+\alpha_{k+1}-1} \exp \left\{-\left(m_{k+1} \gamma s_{k+1}^{-1}+\alpha_{k+1} \mu_{k}^{-1}\right) v\right\} d v=\Gamma\left(m_{k+1} \gamma+\alpha_{k+1}\right)\left(m_{k+1} \gamma s_{k+1}^{-1}+\alpha_{k+1} \mu_{k}^{-1}\right)^{-\left(m_{k+1} \gamma+\alpha_{k+1}\right)},
$$

we have 


$$
p\left(s_{k+1} \mid s_{k}\right)=A_{2} \mu_{k}^{-1}\left(s_{k+1} \mu_{k}^{-1}\right)^{\alpha_{k+1}-1}\left(m_{k+1} \gamma+\alpha_{k+1} s_{k+1} \mu_{k}^{-1}\right)^{-\left(m_{k+1} \gamma+\alpha_{k+1}\right)},
$$

where $A_{2}=A_{1} \Gamma\left(m_{k+1} \gamma+\alpha_{k+1}\right)$. Hence, conditional on $s_{k}, s_{k+1} / \mu_{k}$ follows an $F$-distribution with degrees of freedom $2 \alpha_{k+1}$ and $2 m_{k+1} \gamma$ respectively. This completes the proof.

\section{References}

Bickel, P. J., Chen, C., Kwon, J., Rice, J., Van Zwet, E., Varaiya P., 2007. Measuring traffic. Statistical Science 22(4), 581-597.

Congdon, P., 2001. Bayesian Statistical Modelling. Wiley, London.

Dailey, D. J., 1999. A statistical algorithm for estimating speed from single loop volume and occupancy measurements. Transportation Research part B 33 (5), 313-322.

Gerlough, D. L., Huber, M. J., 1975. Traffic Flow Theory: A Monograph. Transportation Research Board, Washington, D.C.

Haight, F. A., 1963. Mathematical Theories of Traffic Flow. Academic Press, London.

Hall, F. L., Persaud, B. N., 1989. Evaluation of speed estimates made from single-detector data from freeway traffic management systems. Transportation Research Record 1232, Transportation Research Board, National Research Council, Washington, D.C., 9-16.

Hazelton, M., 2004. Estimating vehicle speed from traffic count and occupancy data. Journal of Data Science 2 (3), 231-244.

Jain, M., Coifman, B., 2005. Improved speed estimates from freeway traffic detectors. Journal of Transportation Engineering-ASCE 131(7), 483-495.

Kurkjian, A., Gershwin, S., Houpt, P. Willsky, A., Chow, E., 1980. Estimation of roadway traffic density on freeways using presence detector data. Transportation Science 14 (3), 232-261.

Li, B., 2005. Bayesian inference for origin-destination matrices of transport networks using the EM algorithm. Technometrics 47 (4), 399-408. 
Ljung, L., Soderstrom, T., 1987. Theory and Practice of Recursive Identification. The MIT Press, Cambridge.

Polus, A., 1979. A study of travel time and reliability on arterial routes. Transportation 8 (2), $141-151$.

Salter, R. J., 1989. Highway Traffic Analysis and Design. 2nd ed. MacMillan, London.

Ye, Z. R., Zhang, Y. L., Middleton, D. R., 2006. Unscented Kalman filter method for speed estimation using single loop detector data. Transportation Research Record, 1968, 117125 . 
Table 1. The forgetting factor $\delta$ and estimation error

\begin{tabular}{ccccccccc}
\hline$\delta$ & 0.60 & 0.65 & 0.70 & 0.75 & 0.8 & 0.85 & 0.90 & 0.95 \\
\hline RMSE (mph) & 2.65 & 2.50 & 2.38 & 2.29 & 2.25 & 2.29 & 2.43 & 4.11 \\
\hline
\end{tabular}

Table 2. Average RMSEs (mph) over 30 simulation experiments for the developed method and the classical method

\begin{tabular}{cccccc}
\hline & \multicolumn{2}{c}{ With the 'true' value of EVL } & & \multicolumn{2}{c}{ With an estimate of EVL } \\
\cline { 2 - 3 } \cline { 5 - 6 }$\gamma$ & $\begin{array}{c}\text { The classical } \\
\text { method }\end{array}$ & $\begin{array}{c}\text { The developed } \\
\text { recursive method }\end{array}$ & & $\begin{array}{c}\text { The classical } \\
\text { method }\end{array}$ & $\begin{array}{c}\text { The developed } \\
\text { recursive method }\end{array}$ \\
\hline 15 & 9.5937 & 2.8247 & & 9.5089 & 2.8955 \\
\hline 25 & 7.3644 & 2.5128 & & 7.3558 & 2.5807 \\
\hline
\end{tabular}

Table 3. A comparison of average RMSEs (mph) between Dailey's method and the developed method over 30 simulation experiments

\begin{tabular}{ccc}
\hline$\sigma_{s}$ & $\begin{array}{c}\text { The Dailey's } \\
\text { method }\end{array}$ & $\begin{array}{c}\text { The developed } \\
\text { method }\end{array}$ \\
\hline 14 & 10.7161 & 4.0808 \\
10 & 8.1034 & 3.8196 \\
5 & 4.4396 & 2.8649 \\
\hline
\end{tabular}




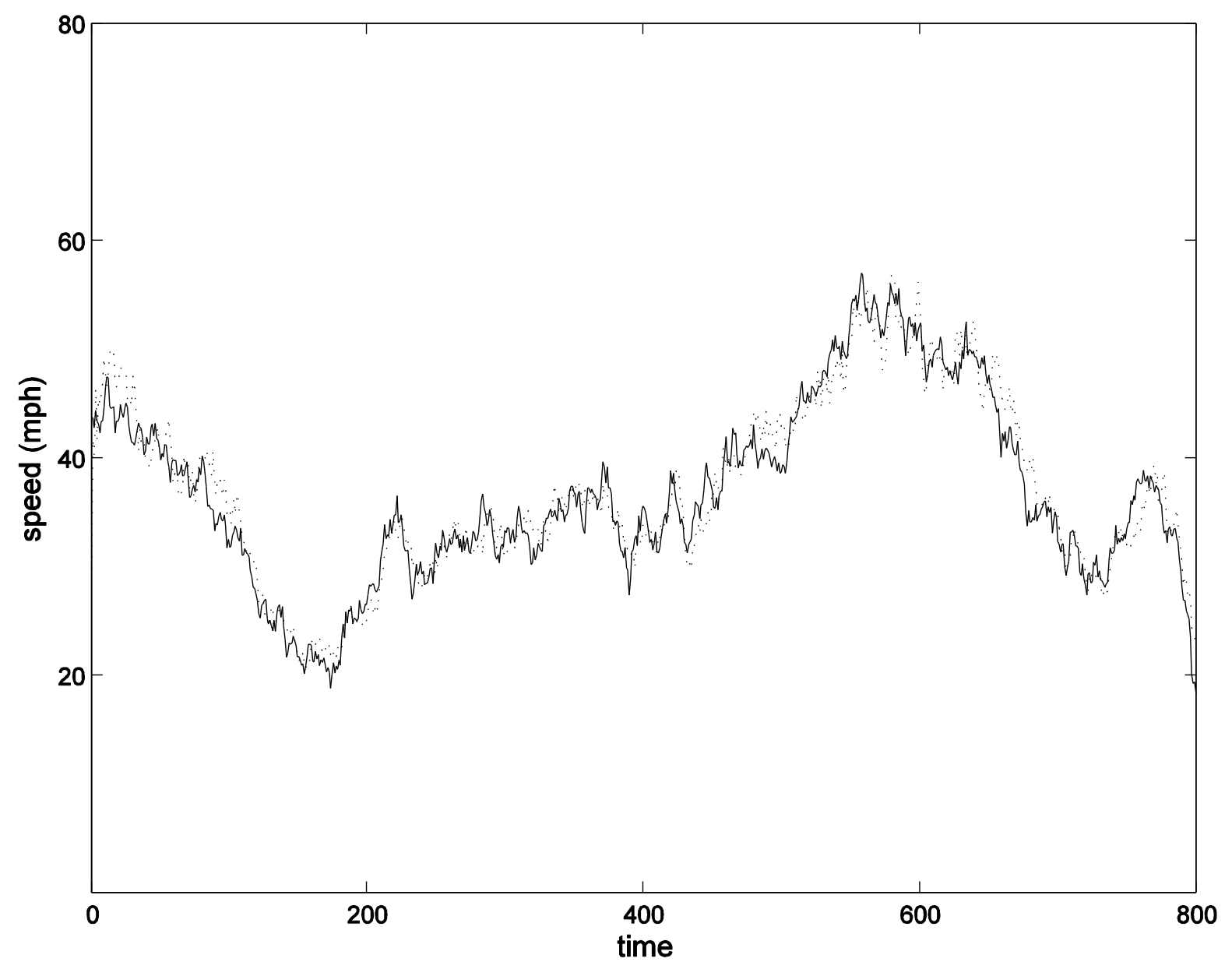

Figure 1a. The 'true' values of vehicular speed (real line) and the estimated values by the recursive method (dotted line). 


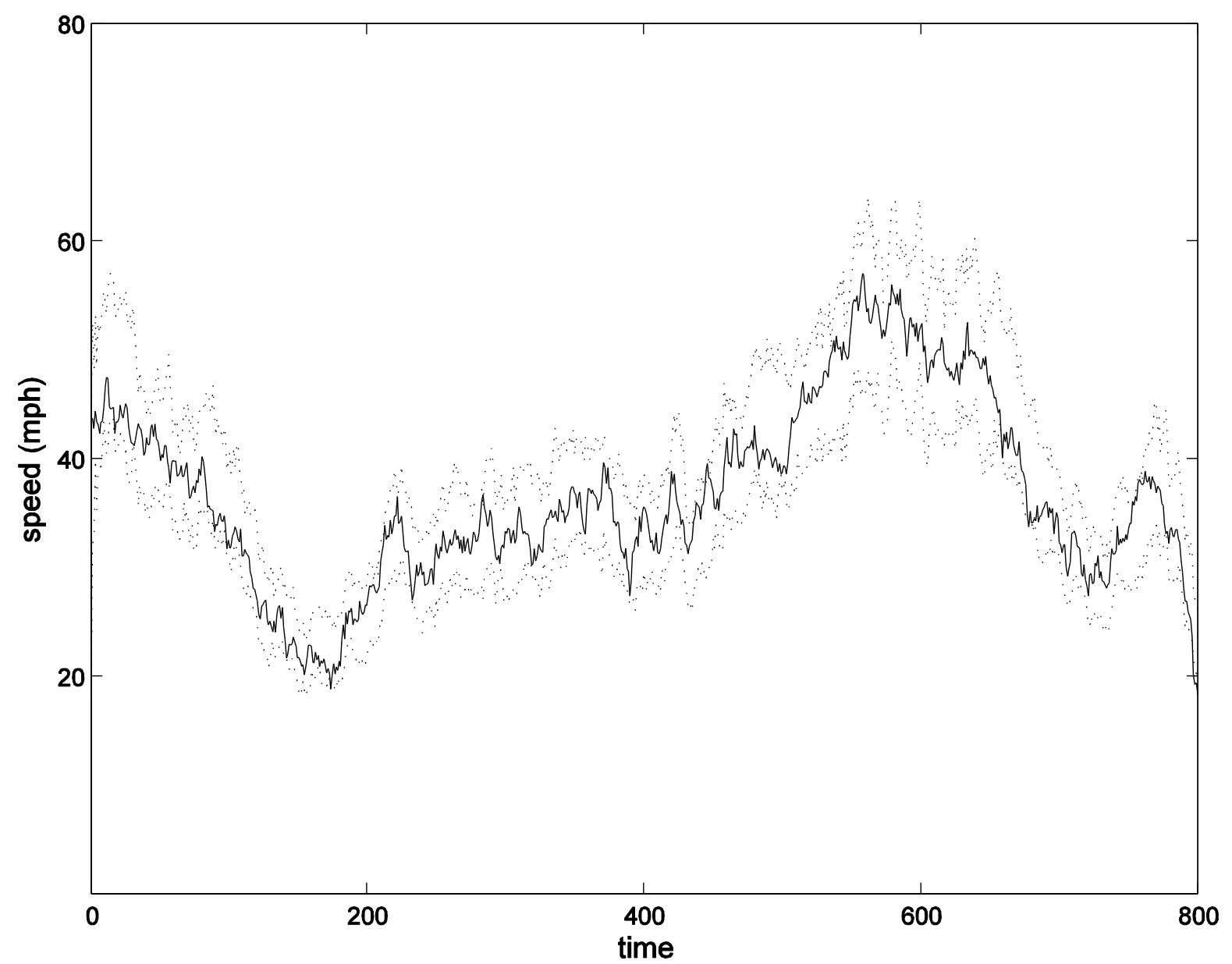

Figure $1 \mathrm{~b}$. The envelop of a nominal 95\% credible intervals (dotted lines) and the 'true' values of vehicular speed (real line). 


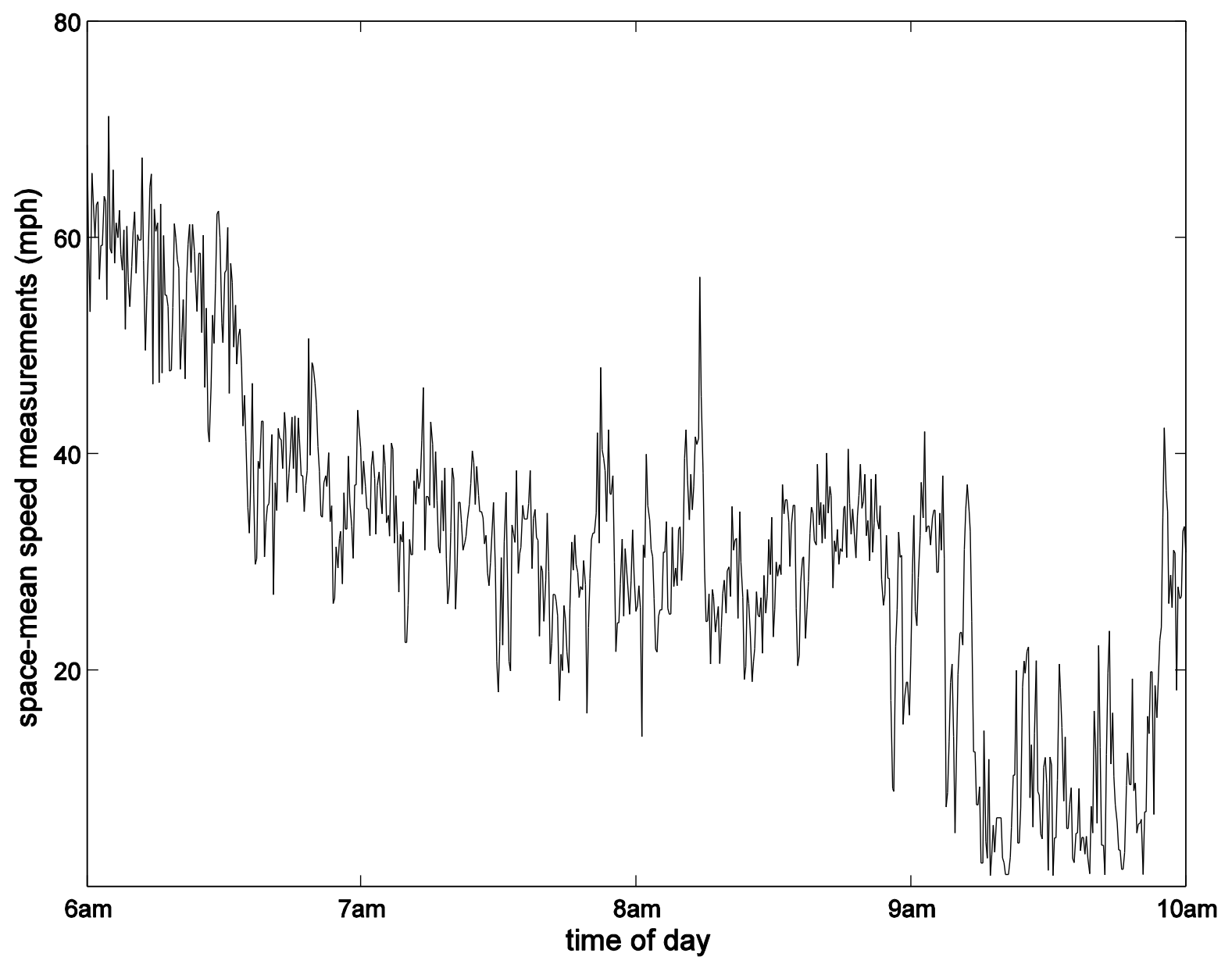

Figure 2a. The observations of space-mean speed. 


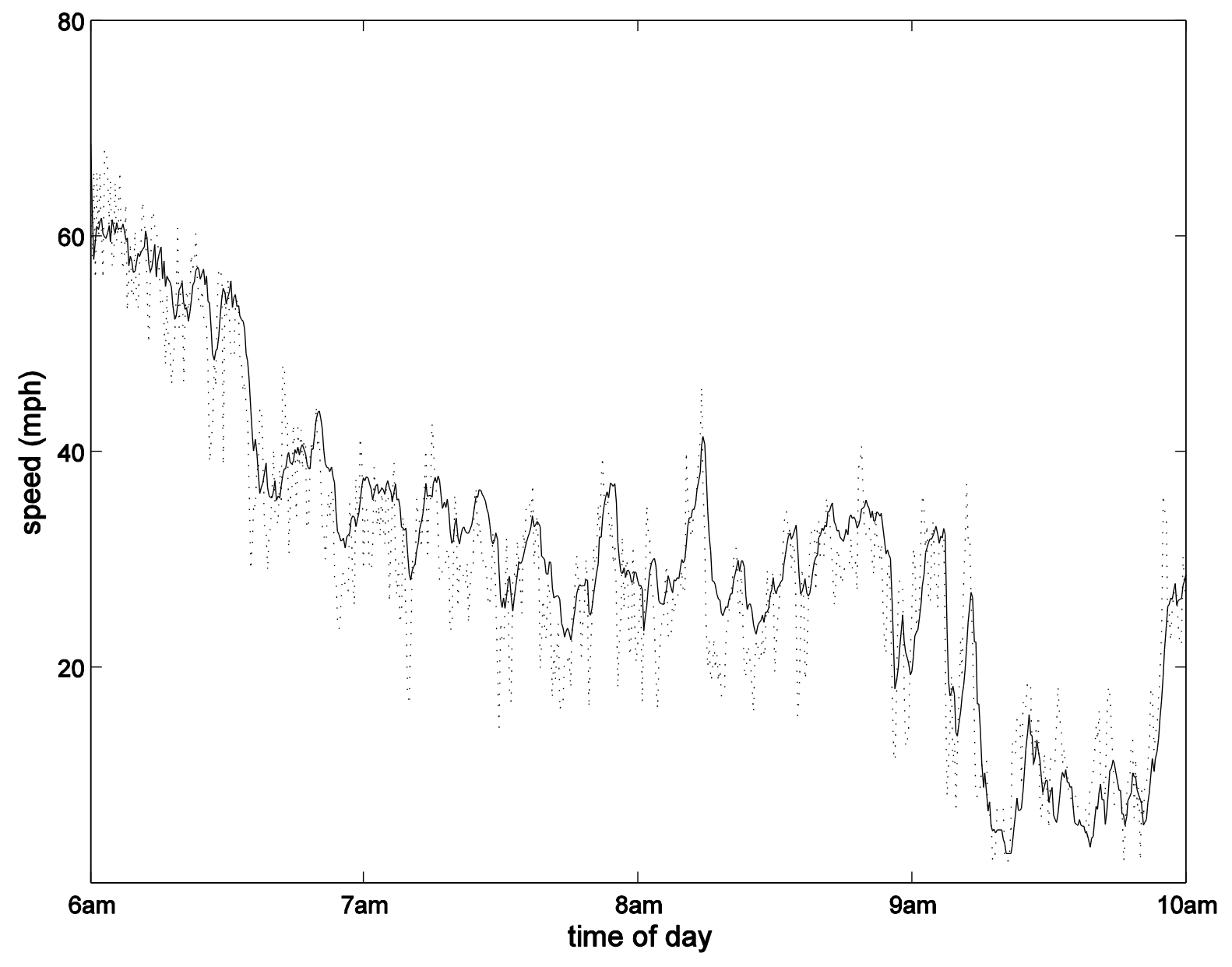

Figure $2 b$. The measured vehicular speed by a speed trap (dotted line) and the estimated vehicular speed by the developed method (real line). 


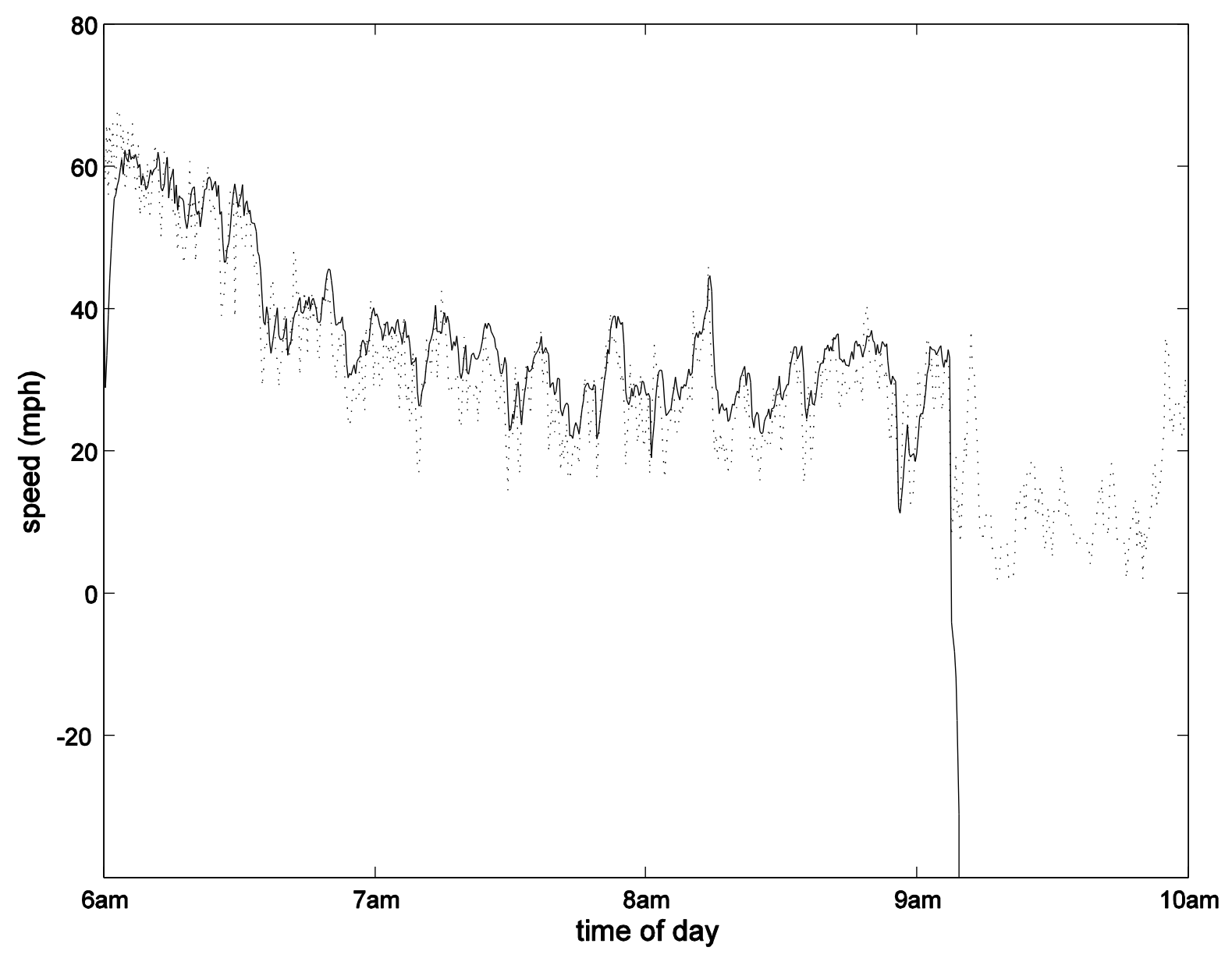

Figure 2c. The measured vehicular speed by a speed trap (dotted line) and the estimated vehicular speed by Dailey' method (real line). 